\title{
Spherical: an iterative workflow for assembling metagenomic datasets
}

Thomas C. A. Hitch ${ }^{1,2^{*}}$ and Christopher J. Creevey ${ }^{1}$

\begin{abstract}
Background: The consensus emerging from the study of microbiomes is that they are far more complex than previously thought, requiring better assemblies and increasingly deeper sequencing. However, current metagenomic assembly techniques regularly fail to incorporate all, or even the majority in some cases, of the sequence information generated for many microbiomes, negating this effort. This can especially bias the information gathered and the perceived importance of the minor taxa in a microbiome.

Results: We propose a simple but effective approach, implemented in Python, to address this problem. Based on an iterative methodology, our workflow (called Spherical) carries out successive rounds of assemblies with the sequencing reads not yet utilised. This approach also allows the user to reduce the resources required for very large datasets, by assembling random subsets of the whole in a "divide and conquer" manner.

Conclusions: We demonstrate the accuracy of Spherical using simulated data based on completely sequenced genomes and the effectiveness of the workflow at retrieving lost information for taxa in three published metagenomics studies of varying sizes. Our results show that Spherical increased the amount of reads utilized in the assembly by up to $109 \%$ compared to the base assembly. The additional contigs assembled by the Spherical workflow resulted in a significant $(P<0.05)$ changes in the predicted taxonomic profile of all datasets analysed. Spherical is implemented in Python 2.7 and freely available for use under the MIT license. Source code and documentation is hosted publically at: https://github.com/thh32/Spherical.
\end{abstract}

Keywords: Assembly, Metagenome, Genomics

\section{Background}

Over the last 10 years, researchers have utilised highthroughput sequencing to investigate the structure and function of microbial communities from diverse environments across the globe [1-3]. While these studies have provided unique and novel insights into the workings of these microbiomes, there is a growing consensus that the tools available are not describing the full functional or taxonomic diversity that the data represents [4]. There is a need for assemblers and workflows to be developed to capture "lost" information and allow the generation of assemblies that represent the entire metagenome sampled.

Mathematically, de novo assembly of a genome falls within the class of problems for which no efficient

\footnotetext{
* Correspondence: thitch@ukaachen.de

'Institute of Biological, Environmental and Rural Sciences (IBERS),

Aberystwyth University, Aberystwyth, Wales SY23 3FG, UK

${ }^{2}$ Functional Microbiome Research Group, Institute of Medical Microbiology, Uniklinik RWTH Aachen, Aachen, Germany
}

algorithm is known (NP-hard) [5], leading to the proposal of a variety of heuristic solutions [6-9]. These have ranged from simple overlap layout consensus approaches (where sequencing reads with overlapping regions are joined together into contigs [5] to more complex approaches such as de Bruijn graphs [10]. Generally, these assemblers have been designed assuming a single genome within the data. However, as sequencing approaches for sampling the genomic information of entire microbial communities (metagenomics) began to emerge [11], it was clear that new approaches may be necessary [12].

By far the biggest issue with metagenomic sequencing datasets is the resulting uneven coverage of the taxa from the microbiome arising from the complexity and uneven distribution of species in natural microbial communities [4]. This leads to over-sequencing of dominant species in the community and heavily fragmented assemblies of the genomes of minority species, if they can be assembled at all [13]. 
Promising solutions to dealing with this problem utilise a 'divide and conquer' approach to partition the data into more easily manageable pieces [13]. For example, the data from environmental samples can be split into "bins" representing different taxa from the community [14]. Sequence reads can be sorted into bins based on properties such as kmer-frequency or the percentage of Guanine and Cytosines (GC) they contain [15]. This has the potential to increase the assembly rate of low abundance species, however it depends heavily on accurate partitioning of the data [16]. Indeed, bins of metagenomic data produced in this manner may represent a single species or an entire phylum depending on the complexity of the community [16]. SLICEMBLER [8] also implements a 'divide and conquer' approach by generating pre-determined "slices" of the metagenome based on coverage. Each slice is then assembled separately and frequently occurring strings are identified and used to merge contigs. This works well for deeply sequenced genomic datasets where coverage is known, however in metagenomics datasets from uncharacterised microbial communities, coverage is generally an unknown variable [17]. Previous attempts at utilising iterative binning for assembly have been developed but are not publically available [18].

Digital normalization is another commonly utilized approach for tackling complex datasets. This method, implemented in the Khmer package [19], uses kmers to identify and reduce the occurrence of highly duplicated portions of the data. This has the effect of reducing coverage of over-represented taxa, and normalising the coverage to make it more even [19]. However, while this pre-processing step allows for reduction of the datasets size, it does not reduce the complexity of the data [19] leaving subsequent assemblies prone to the problem of under-representing sections of the community.

We propose a simple but effective heuristic to address this problem that can be used in conjunction with any of the above-mentioned assembly approaches. Based on an iterative approach, our workflow (called Spherical) identifies the reads not accounted for in an assembly, and uses them as input for successive rounds of assemblies. Spherical also provides the option to minimize the resources required by assembling random subsets of the whole in a "divide and conquer" manner. The inherent downside to this approach is that by running multiple iterations of assembly, the time requirements are also increased.

\section{Methods}

\section{Implementation}

\section{Spherical workflow}

Spherical uses an iterative workflow that identifies the raw data not represented in an initial assembly. This is then used as input for a subsequent assembly. This process is repeated until a pre-defined cut-off is reached, or until all the data is incorporated. The workflow is summarised in Fig. 1.

The Spherical workflow is composed of 5 steps:

Step 1: Sub-sample selection

The first step in Spherical is the optional initial subsampling of the sequencing data (Fig. 1.2). This can be advantageous when working with very large datasets as it reduces the complexity of the assembly, reducing memory and time requirements. In this process a random sub-sample (defined by the option '- $R$ ') is taken from the input sequencing data. Using a sub-sample size of ' 1 ' selects the entire input dataset instead. If only one value is given to '-R', then Spherical will apply this sub-sample fraction at every iteration, however the user also has the option of providing multiple different values to be used at each iteration.

\section{Step 2: Assembly}

The sub-sample is then assembled using the assembler of choice (Fig. 1.3). By default, Spherical uses either Velvet [6] or ABySS [20] for assembly, it is easy to extend Spherical to utilise other assemblers also.

\section{Step 3: Alignment}

When the assembly is completed, Spherical uses Bowtie 2 [21] to align all reads previously unaligned (in iteration 1 , this is all the reads) to the contigs resulting from the assembly. All reads that do not align to the assembly produced at this iteration are saved for subsequent rounds. If a read aligns to the assembly at this point, it is considered utilised and hence excluded from the subsequent iterations. Specific commands can be passed to Bowtie2 using the '-u' command followed by the commands, for example $-\mathrm{u}$ “-N $3-\mathrm{k} 2$ " would pass the commands to Bowtie 2 to set the maximum number of mismatches in the seed to 3 and to look for at most 2 distinct valid alignments for each read.

\section{Step 4: Assessment}

The user can define two parameters to be used by Spherical to determine the completeness of the assembly. The first is based on the number of iterations completed ('-iter'). When Spherical has completed all iterations defined by this value, it will halt and move to Step 5. The second option is based on the proportion of reads utilised by the total assembly ('-align 0100 '). This is calculated as the number of reads currently unaligned, divided by the total number of reads initially provided, multiplied by 100 . When Spherical determines that the predetermined alignment rate has been reached, it will halt carrying out iterations and move to Step 5. The default options are 5 iterations or an alignment rate of $70 \%$. If neither criteria has been 


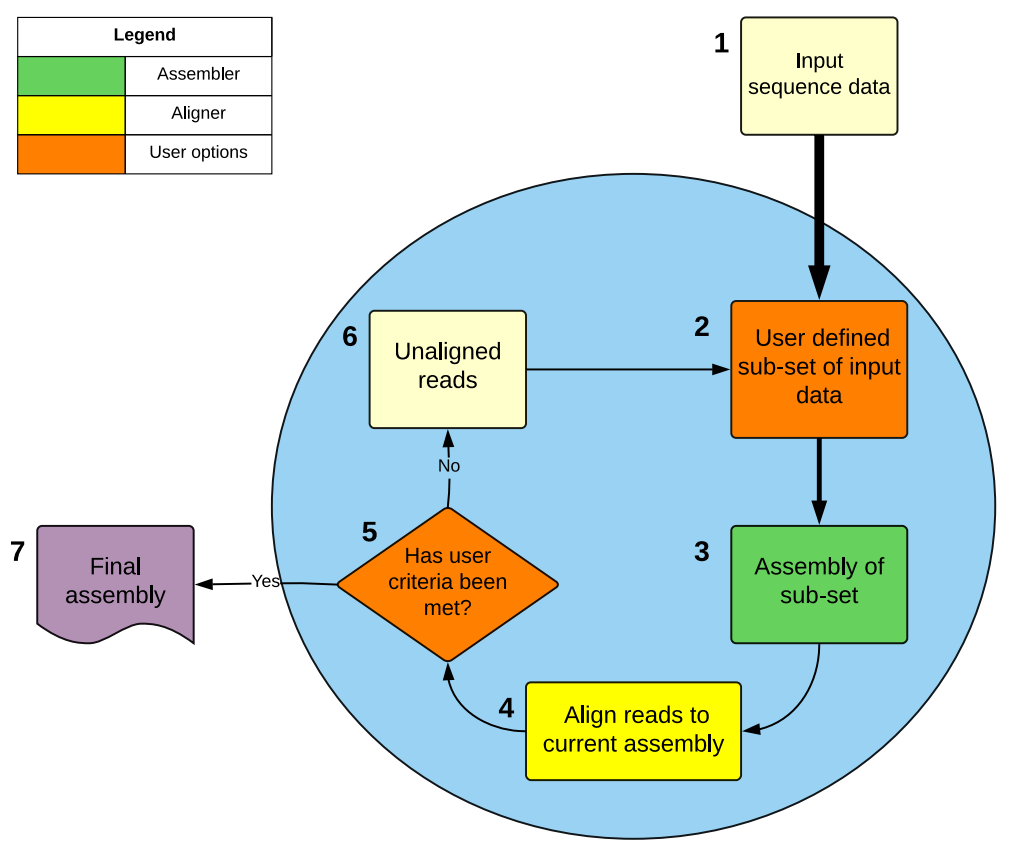

Fig. 1 A flow-chart of the steps used by Spherical. The blue circle encompasses all processes that are carried out in an iterative manner until the results meet the 'user defined criteria'. The 'user defined criteria' is defined as any user option which indicates a point at which Spherical should stop iterating. The arrows width indicates the possible decrease in file size depending on user sub-sample selection. 1; User input data (usually quality controlled sequencing files). 2; Spherical takes a random subset of the input sequencing data. The size of the subset is determined by user. 3; An assembly of the subset is generated. 4; The number of reads aligning to the combined assembly are determined. 5; If the number of reads aligning meets the user criteria Spherical will move to step 7, otherwise Spherical will continue to step 6. 6; Reads that do not align to the combined assembly are used as input for the next round. 7; Spherical exits and combines the individual iterations assemblies into a single file

met, Spherical will pass all unaligned reads to step 1 for another iteration of assembly.

\section{Step 5: Final output}

Once any of the user-defined criteria for halting have been met, Spherical will combine the assembly from each iteration into a single file. Assembly statistics such as N50, lengths of longest and shortest contigs, the standard deviation of the lengths and the alignment rate are calculated for each iteration and for the combined assembly file. Using the '-m' option merges the contig files produced by each iteration into a single file for ease of use. A final assembly which attempts to combine all the contigs created, can be carried out by specifying the '-f' option.

To illustrate the outputs of the Spherical workflow, we carried out assemblies of both simulated and real metagenomics datasets. These are presented in the following sections.

\section{Simulated metagenome}

The simulated metagenome examined came from a previous study [22]. This consisted of 400 species (1 genome per species) with abundances varying from $0.66 \%$ to $0.22 \%$. This dataset was originally produced to study the effect of sequencing technologies on the assembly of metagenomic datasets. The Spherical assembly of this dataset used Velvet with a kmer of 31 and subsample size of 1 (the entire dataset).

\section{Real metagenomic datasets}

We also present the results from the application of Spherical to three published metagenomic datasets: chicken caecum [23]; human oral cavity [24] and groundwater from the Yucatan peninsula (Table 1). All three metagenomic datasets were obtained from MGRAST [25]. The Chicken cecum dataset was selected due to having a low sequencing depth and therefore allowing comparison of assembly methods on a small dataset [23]. The human oral cavity dataset was selected due to being a complex microbiome and providing moderate sequencing depth [24]. The Yucatan groundwater dataset was selected due to containing a complex microbiome and providing a high level of sequencing depth.

Table 1 Information on each metagenomics dataset tested using Spherical

\begin{tabular}{lll}
\hline Environment & Dataset size (Gbp) & MGRAST project ID \\
\hline Chicken Caecum [22] & 0.06 & 101 \\
Human oral cavity [23] & 0.63 & 128 \\
Yucatan groundwater & 29.00 & 5969 \\
\hline
\end{tabular}

For each metagenomic dataset the table states the source environment, the datasets size in Giga base pairs (Gbp) and its MGRAST project ID 


\section{Methods of assembly}

To ensure consistency, Velvet was used to assemble each of the above datasets. Initially, an optimal kmer size was determined for each datasets by carrying out multiple initial assemblies using the entire dataset with kmer sizes of 21,31,41 and 51. Alignments of the raw reads to each of these initial assemblies was then carried out using Bowtie2 (with options: $-\mathrm{N} 1$ ) and the number of reads aligning determined. For each dataset, the kmer size that resulted in the largest fraction of reads aligning was used as the optimal kmer for all further assemblies of that dataset (Additional file 1: Table S1).

Following determination of the optimal kmer size, each of the datasets were assembled using three approaches: 1) An assembly using only velvet; (basic assembly); 2) Initial digital normalization of the reads using Khmer, followed by an assembly with Velvet (Normalized assembly); and 3) Assembly with the Spherical workflow, using Velvet as the assembler (Spherical Assembly). These were conducted with the entire dataset (sub-set $=1$ ).

The settings used with Velvet were the same for all three approaches using the kmer sizes determined earlier and the '-exp_cov auto' option to allow Velvet to calculate the expected coverage. Meta-Velvet is a metagenomic assembler, which acts as a final step in standard Velvet assembly and so was initially investigated using the 'meta-velvetg' command on the output from Velvet. The options for running Spherical on each dataset are shown in Additional file 1 Table S5. Each assembly ran for 5 iterations apart from the chicken caecum dataset for which an optimal assembly was achieved following iteration 2 .
Digital normalisation was included as a comparative approach due to its ability to reduce the size of a dataset by removal of reads consisting of redundant kmers, while keeping the datasets original complexity by retaining reads including unique kmers [19]. Digital normalization was applied using the "normalize-by-median.py" script of Khmer, with a kmer of 20, 4 hash tables of size $32 \mathrm{e}^{9}$ and an ideal median of 20, as suggested by the Khmer manual.

The amount of RAM necessary to carry out an assembly is a limiting factor for many large metagenomics assemblies, requiring workarounds, such as kmer normalization. For this reason the amount of RAM utilised during each assembly was monitored (Table 2).

\section{Assembly quality}

We used a simulated metagenomic dataset generated from 400 genomes [22] to assess the quality of the de novo assemblies. Contig scores [22] were calculated by identifying the highest scoring (bitscore) match to each contig in the 400 genomes using BLASTN [26]. The formula: $\frac{\text { coverage percentage }}{100} \times$ percentage identity was used to calculate the contig score ranging from 0 to 100 . A contig score of 100 indicates the entire contig precisely matches a region from the original genome and 0 means there was no matching region [22].

Contig scores cannot be calculated for real metagenomic assemblies due to requirement for a having an assembled genomes for comparison. Instead, for these datasets standard assembly statistics were calculated to provide insight in the quality of each assembly; alignment rate (defined as the percentage of sequenced reads

Table 2 Assembly statistics comparing dataset assemblies for each method

\begin{tabular}{lllllll}
\hline Dataset & Method & RAM usage (Gb) & Alignment (\%) & False bases (\%) & Longest contig & Number of contigs \\
\hline Cecum & Normalised & 119 & 29.5 & 0.01 & 831 & 103,618 \\
& Base assembly & 1 & 29.5 & 0.01 & 831 & 103,618 \\
& Metavelvet & 2 & 29.1 & 0.07 & 831 & 103,618 \\
& Spherical (1) & 2 & 30.9 & 0.04 & 831 & 138,995 \\
Oral & Normalised & 14 & 8.1 & 0.01 & 3337 & $1,825,177$ \\
& Base assembly & 25 & 13.0 & 0.02 & 4548 & $1,178,611$ \\
& Metavelvet & 15 & 13.0 & 0.07 & 4548 & $1,178,611$ \\
Ground water & Spherical (1) & 5 & 24.6 & 0.19 & 2380 & $1,053,802$ \\
& Normalised & 361 & 52.8 & 3.86 & 117,274 & $5,721,819$ \\
& Base assembly & 376 & 52.0 & 3.84 & 117,274 & $5,772,465$ \\
& Metavelvet & 376 & 52.0 & 4.04 & 117,274 & $5,772,461$ \\
& Spherical (1) & 377 & 59.7 & 2.89 & 117,274 & $13,312,643$ \\
& Spherical (0.25) & 129 & 51.5 & 3.50 & 104,353 & $7,851,021$ \\
& Spherical (0.033) & 107 & 49.8 & 3.78 & 53,836 & $7,145,998$ \\
\hline
\end{tabular}

The first column indicates the dataset utilized whilst the second column identified the assembly methodology. To identify the different subsampling amounts during each Spherical assembly the subsample size is stated in brackets in the method column. The final 5 columns provide information on the computational needs for each assembly (RAM usage) as well as statistics about the produced assemblies e.g. number of contigs and alignment (\%) 
which align to the assembly), N50 (defined as the contig length where $50 \%$ of the entire assembly is contained in contigs of equal or longer length the N50 value) and "false base rate" (defined as the percentage of bases in the assembly to which no read aligns). To calculate the "false base rate" the raw data from each dataset was aligned to each of its subsequent assemblies using Bowtie2 [21] with the option "-n 1", which allows for a single mismatch in the seed region.

\section{Taxonomic identification}

For all assemblies the best taxonomic matches for each contig was identified from the Bacterial and Archaeal subsets of the UNIPROT database (downloaded 10/ 2014) using RAPsearch (with settings: bitscore $>=40$ ) $[27,28]$. All hits found in the database were converted into a general feature format (GFF) file using the "blast2gff" command in MGKIT (http://mgkit.readthedocs.io/en/latest/) and subsequently filtered for the top non-overlapping hits with the "filter-gff" command (settings; -s 100) from MGKIT. This has the effect of resulting in a single best taxonomic match for each contig. The HTSeq-count (settings; $-m$ intersection-nonempty) command from the HTSeq package [29] was used to identify the number of reads assigned to phyla within each assembly.

\section{Sub-sampling data with Spherical}

Spherical allows a user to specify a subset of data to be used for each round of assembly. This is useful for the analysis of very large metagenomic datasets when RAM usage or length of time of the analysis can become issues. To demonstrate this we carried out 3 different assemblies with different sub-sampling sizes on the largest metagenomic dataset analysed (from the Yucatan Peninsula). 1) Using all the reads (sub-sample size $=1$ ), 2) A random subsample of a quarter of all the reads (subsample size $=0.25)$ and 3) A random subsample of one thirtieth of the reads (set-sample size $=0.033$ ). Each assembly was allowed to run for five iterations (where the same subsample size was utilised in each iteration) with the following settings: -k 41 -align 99 -iter 5 . These assemblies were compared using their alignment rate, RAM usage, N50, false base rate and longest contig size.

\section{Effect of multiple iterations of assembly}

The biodiversity within each iteration of assembly was identified and assessed for the caecum, oral and Yucatan groundwater datasets. To test if each iteration from a single dataset returned similar taxonomic profiles, a Chi-square test for homogeneity was conducted. A Ztest was then conducted between iterations which exhibited significant changes in their taxonomic profiles in order to identify which specific taxonomic groups altered in abundance. The $p$-values were then corrected using the Benjamani-Hochberg method.

\section{Results}

\section{Quality analysis of resulting assemblies}

We used a simulated metagenomic dataset [22] created from 400 species of varying abundance to investigate the accuracy of contigs produced by the Spherical workflow. For each assembled contig, within each iteration, a 'contig score' [22] was calculated which represents the accuracy of the reconstruction. We found that over $90 \%$ of the contigs reconstructed by Spherical had a contig score $>=95$, representing that they were $95 \%$ identical to the original genome from which the reads were produced. Furthermore, fewer than $1 \%$ of the contigs had a score less than 50 (see Additional file 1: Table S3). The secondary iterations carried out also allowed alignment of an additional $5.25 \%$ of the raw reads compared to the base assembly. The proportion (\%) of reads assigned to each genome within the dataset, base assembly and Spherical assembly are provided in Additional file 1: Table S4.

\section{Capture of additional information}

The smallest of the microbiome datasets analysed (0.06Gbp from the Chicken Caecum) resulted in $29.5 \%$, $29.5 \%$, 30.9\% alignment rate for the base, normalized and Spherical assembly respectively (Table 2). While alignment rates were very similar for the three assembly approaches used Spherical utilised 1.4\% more of the raw reads than the other approaches but at the cost of slightly lowering the N50 (from 109 to 104) and increasing the false base rate (from $0.01 \%$ to $0.04 \%$ ).

The medium-sized human oral cavity dataset $(0.63$ Gbp), resulted in greater variability in how the different assembly approaches performed. Spherical increased the alignment rate (from 13\% to 24.6\%) and N50 (from 190 to 234) compared to the next best approach (base assembly) (Table 2). However, the false base rate also increased slightly (from $0.02 \%$ to $0.19 \%$ ) indicating the Spherical assembly was including a small number of bases without evidence supporting their inclusion from the raw reads.

Finally, for the largest datasets analysed (29 Gbp from Yucatan groundwater), Spherical resulted in an increased alignment rate (from $52.8 \%$ to $59.7 \%$ ) and a decreased false base rate (from $3.86 \%$ to $2.89 \%$ ). However, the N50 was reduced (from 330 to 211) in comparison to the normalised assembly. Within these datasets Meta-velvet continually produces assemblies with an increased number of false bases and was unable to outperform the base assembly, therefore meta-velvet was not investigated further. 


\section{Sub-sampling the sequencing data}

The effect of altering the sub-sample size was tested on the largest of the metagenomics datasets (Yucatan groundwater). As shown in Additional file 1: Table S5, the change in sub-sample size caused a small reduction in the quality of the resulting assembly; decreasing the false base rate from $3.86 \%$ to $3.78 \%$, reducing the N50 from 330 to 189 and slightly reducing the alignment rate from $52.8 \%$

a
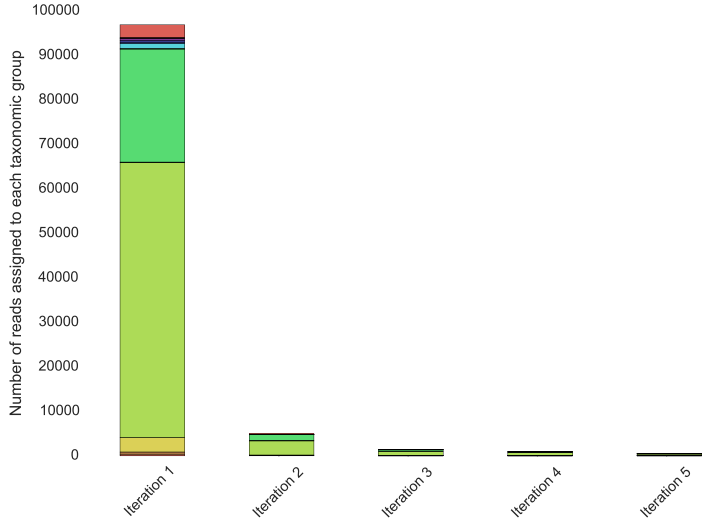

b

Iteration

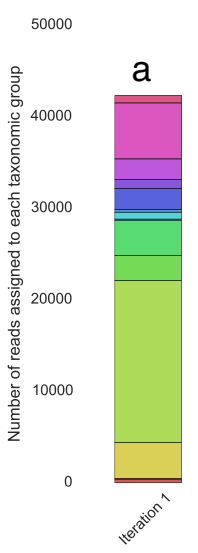

b
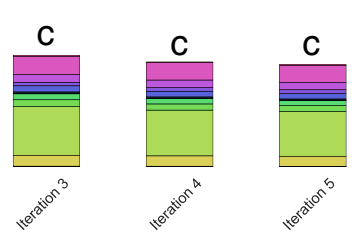

C
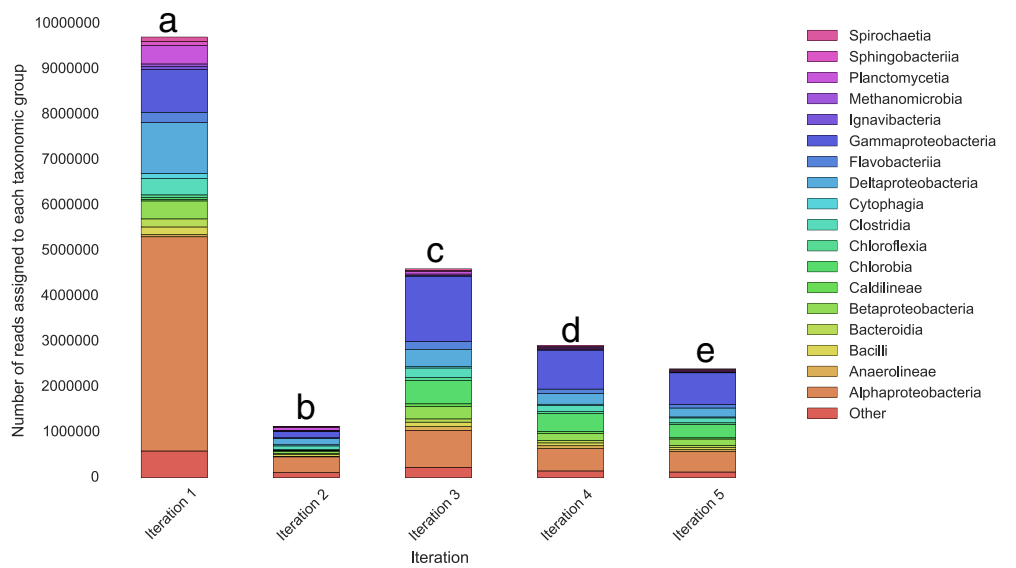

Fig. 2 Taxonomic breakdown of each iteration for the chicken ceacum (a), human oral cavity (b) and Yucatan groundwater Spherical (sub-sample size $=1)(\mathbf{c})$ assemblies at the class level. Each bar represents the number of reads that could be assigned to a taxonomic Class within the assembly from each iteration. The colours represent different taxonomic Classes identified in the legend on the right. The letters represent the results of the significance tests where bars with the same letter are not significantly different according to the Chi $^{2}$ test for homogeneity 
to $49.8 \%$ when compared to the base assembly. However, the taxonomic profile estimated from the resulting assemblies remained unchanged (Additional file 1: Figure S1). The effect of random sampling of reads for each iteration was investigated by assembling the Oral dataset 5 times with the same options ( 5 iterations, $\mathrm{kmer}=31$, subset size $=5)$. The alignment rate of both the final assemblies and individual iterations showed no variation $( \pm 0.0 \%)$ across the 5 runs.

\section{Multiple iterations of assembly}

The secondary iterations in Spherical utilise previously unaligned sequencing information to assemble new genomic regions, creating more contigs for analysis (Fig. 2). The Spherical assemblies also had an increased number of reads aligning to annotated genes $(11 \%, 109 \%$ and $43 \%$ in the chicken caecum, human oral and Yucatan groundwater dataset respectively (Additional file 1: Table S6)).

Whilst the taxonomic profile for the chicken caecum was consistent between iterations (Fig. 2a), we observed a significant change in between iterations both in the human oral (Fig. 2b) and Yucatan groundwater datasets $(P<0.05$, Chi2 test for homogeneity) (Fig. 2c). The taxonomic profiles of each iteration of assembly for the Yucatan groundwater dataset was unique (Fig. 2c), suggesting novel genomic regions were being included within each iteration, increasing the accuracy of the final taxonomic composition. A two-proportion Z-test (Benjamini-Hochberg corrected) was then used to identify which taxonomic groups showed a change in abundance in the secondary iterations compared to the base assembly. The inclusion of the additional genomic regions Spherical assembled in the secondary iterations caused a significant change in the taxonomic profile of each dataset compared to both the base assembly and Digital normalisation (Additional file 1: Figure S1).

\section{Discussion}

We investigated the quality of the assemblies from Spherical using a simulated metagenome and confirmed accurate assembly of genomic regions without a decrease in quality (Additional file 1: Table S3). Furthermore with real metagenomic datasets the secondary iterations resulted in up to $109 \%$ more reads aligning to annotated genes compared to the base assembly (Additional file 1: Table S6). This resulted in significant changes in the overall taxonomic profile predicted for both the human oral cavity $(P<0.05)$ and Yucatan groundwater metagenomes $(P<0.05)$. Unsurprisingly, the contigs from the subsequent iterations of assembly were more fragmented, resulting in a lower N50, however they represented information that would otherwise have been missed if a single round of de novo assembly had been used.
The sub-sampling option of Spherical results in a reduction of up to $70 \%$ in the RAM required to assemble the metagenomes with only a small reduction in alignment rate compared to the base assembly (Table 2). This will be attractive to research groups who lack access to sufficient computational facilities to carry out assemblies of complex metagenomes in single step. The inherent downside to this approach is that by running multiple iterations of assembly, the time requirements are also increased.

\section{Conclusion}

Spherical is a workflow that allows the capture and use of data that might otherwise be missed in a metagenomics dataset assembly. It allows the construction of high quality assemblies of metagenomics datasets without restricting users to particular tools or assembly approaches. Implemented in Python it is simple to use and freely available to the scientific community under an MIT license. If applied to novel or previously published datasets it provides the opportunity to reveal novel biological information that may otherwise have been missed.

\section{Additional file}

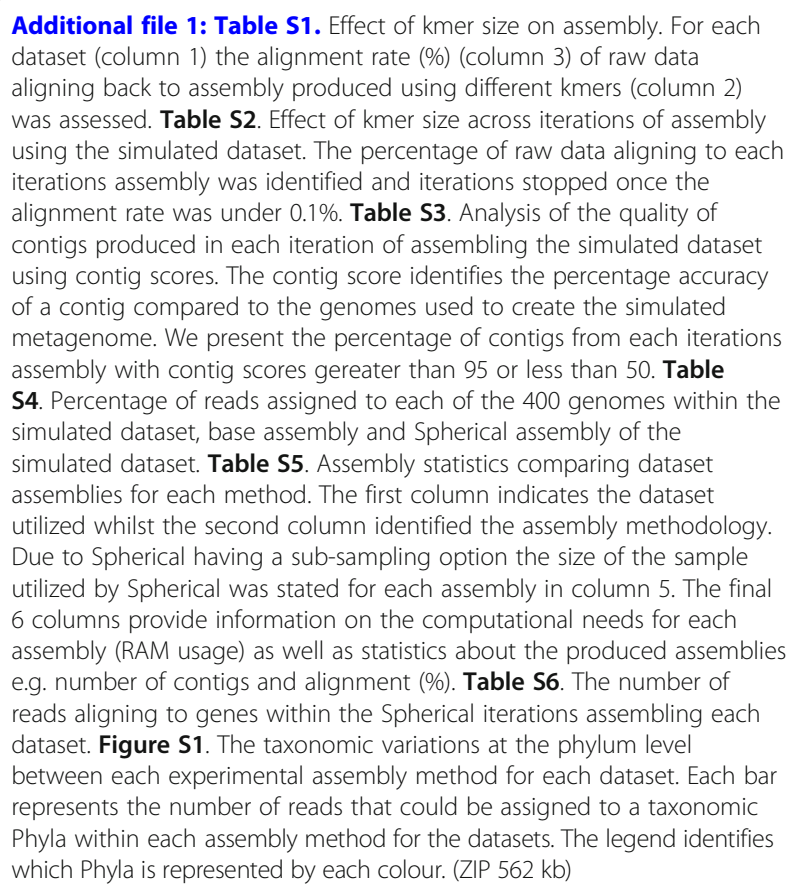

Acknowledgements

We wish to thank the advice provided by Francesco Rubino to this project.

\section{Funding}

TCAH was funded through the New Zealand fund for Global Partnerships in Livestock Emissions Research (GPLER). CJC was funded under the Biotechnology and Biological Sciences Research Council (BBSRC) Institute Strategic Programme Grant, Rumen Systems Biology, (BB/E/W/10964A01). 


\section{Availability of data and materials}

All datasets analysed during this study are available on the MG-RAST webserver using the project IDs provided in Table 1. Spherical is implemented in Python 2.7 and freely available for use under the MIT license. Source code and documentation is hosted publically at: https://github.com/thh32/Spherical

\section{Authors' contributions}

TCAH wrote Spherical and conducted all research in this paper while a PhD student under CJC. CJC guided the research and edited this paper. Both authors have read and approve the publication of this manuscript.

\section{Ethics approval and consent to participate}

Not applicable

\section{Consent for publication}

Not applicable

\section{Competing interests}

The authors declare that they have no competing interests.

\section{Publisher's Note}

Springer Nature remains neutral with regard to jurisdictional claims in published maps and institutional affiliations.

Received: 6 June 2017 Accepted: 16 January 2018

Published online: 24 January 2018

\section{References}

1. Krohn-Molt I, et al. Metagenome survey of a multispecies and algaassociated biofilm revealed key elements of bacterial-algal interactions in photobioreactors. Appl Environ Microbiol. 2013;79:6196-206.

2. van der Lelie $D$, et al. The metagenome of an anaerobic microbial community decomposing poplar wood chips. PLoS One. 2012;7:e36740.

3. Modi SR, Lee HH, Spina CS, Collins JJ. Antibiotic treatment expands the resistance reservoir and ecological network of the phage metagenome. Nature. 2013;499:219-22.

4. Nagarajan N, Pop M. Sequence assembly demystified. Nat Rev Genet. 2013; 14:157-67.

5. Medvedev, P., Georgiou, K., Myers, G. \& Brudno, M. Computability of Models for Sequence Assembly. 289-301 (2007).

6. Namiki T, Hachiya T, Tanaka H, Sakakibara Y. MetaVelvet: an extension of velvet assembler to de novo metagenome assembly from short sequence reads. Nucleic Acids Res. 2012;40:e155

7. Boisvert S, Raymond F, Godzaridis E, Laviolette F, Corbeil J. Ray meta: scalable de novo metagenome assembly and profiling. Genome Biol. 2012; 13:R122.

8. Mirebrahim H, Close TJ, Lonardi S. De novo meta-assembly of ultra-deep sequencing data. Bioinformatics. 2015:31:i9-i16.

9. Conway T, Wazny J, Bromage A, Zobel J, Beresford-Smith B. Gossamer-a resource-efficient de novo assembler. Bioinformatics. 2012;28:1937-8.

10. Compeau PEC, Pevzner PA, Tesler G. How to apply de Bruijn graphs to genome assembly. Nat Biotechnol. 2011;29:987-91.

11. Venter JC, et al. Environmental genome shotgun sequencing of the Sargasso Sea. Science. 2004;304:66-74

12. Lai B, Ding R, Li Y, Duan L, Zhu H. A de novo metagenomic assembly program for shotgun DNA reads. Bioinformatics. 2012;28:1455-62.

13. Bergeron A, Belcaid M, Steward GF, Poisson G. Divide and Conquer: Enriching Environmental Sequencing Data. 2007; https://doi.org/10.1371/ journal.pone.0000830.

14. Mohammed MH, Ghosh TS, Singh NK, Mande SS. SPHINX-an algorithm for taxonomic binning of metagenomic sequences. Bioinformatics. 2011;27:22-30.

15. Dro, J. \& Mchardy, A. C. axonomic binning of metagenome samples generated by next-generation sequencing technologies. (2012);13: 646-655.

16. Wang Y, Leung HCM, Yiu SM, Chin FYL. MetaCluster 5.0: a two-round binning approach for metagenomic data for low-abundance species in a noisy sample. Bioinformatics. 2012;28:i356-62.

17. Peng Y, Leung HCM, Yiu SM, Chin FYL. IDBA-UD: a de novo assembler for single-cell and metagenomic sequencing data with highly uneven depth. Bioinformatics. 2012;28:1420-8.
18. Kim, M. et al., 2013. MetaPar: metagenomic sequence assembly via iterative reclassification. 2013 IEEE Global Conference on Signal and Information Processing, GlobalSIP 2013 - Proceedings, pp.43-46.

19. Crusoe, M. R. et al. The khmer software package : enabling efficient nucleotide sequence analysis [ version 1; referees : 2 approved, 1 approved with reservations ]. 1-12 (2015). https://doi.org/10.12688/f1000research.6924.1.

20. Simpson, J. T., Wong, K., Jackman, S. D., Schein, J. E. \& Jones, S. J. M. ABySS : A parallel assembler for short read sequence data. 1117-1123 (2009). https://doi.org/10.1101/gr.089532.108.

21. Langmead B, Salzberg SL. Fast gapped-read alignment with bowtie 2. Nat Methods. 2012:9:357-9.

22. Mende DR, et al. Assessment of metagenomic assembly using simulated next generation sequencing data. PLoS One. 2012;7:e31386.

23. Qu A, et al. Comparative metagenomics reveals host specific Metavirulomes and horizontal gene transfer elements in the chicken cecum microbiome. PLoS One. 2008;3:19.

24. Belda-Ferre $P$, et al. The oral metagenome in health and disease. ISME J. 2012;6:46-56.

25. Meyer $\mathrm{F}$, et al. The metagenomics RAST server - a public resource for the automatic phylogenetic and functional analysis of metagenomes. BMC Bioinformatics. 2008:8:1-8.

26. Altschul SF, Gish W, Miller W, Myers EW, Lipman DJ. Basic local alignment search tool. J Mol Biol. 1990;215:403-10.

27. Wu CH, et al. The Universal Protein Resource ( UniProt ): an expanding universe of protein information. Nucleic Acids Res. 2006;34:187-91.

28. Zhao $Y$, Tang $H$, Ye $Y$. RAPSearch2: a fast and memory-efficient protein similarity search tool for next-generation sequencing data. Bioinformatics. 2012;28:125-6.

29. Anders S, Pyl PT, Huber W. Genome analysis HTSeq - a Python framework to work with high-throughput sequencing data. Bioinformatics. 2015;31: $166-9$

\section{Submit your next manuscript to BioMed Central} and we will help you at every step:

- We accept pre-submission inquiries

- Our selector tool helps you to find the most relevant journal

- We provide round the clock customer support

- Convenient online submission

- Thorough peer review

- Inclusion in PubMed and all major indexing services

- Maximum visibility for your research

Submit your manuscript at www.biomedcentral.com/submit
Biomed Central 